\title{
Textbook Economics: Zur Wissenschaftssoziologie eines wirtschaftswissenschaftlichen Genres
}

\section{Einleitung: Wirtschaftswissenschaftliche Lehrbuchliteratur als Sozialisationsmedium und hegemoniale Deutungsinstanz}

Von Paul Samuelson, dem bekannten neoklassischen Ökonomen und Autor eines der erfolgreichsten einführenden wirtschaftswissenschaftlichen Lehrbücher ist die Aussage überliefert: „I don't care who writes a nation's laws - or crafts its advanced treaties - if I can write its economics textbooks" (zit. n. Nasar 1995). Das Zitat gibt einen ersten Hinweis auf die Bedeutung und Reichweite, die ökonomische Lehrbuchliteratur spätestens im Zuge der disziplinären Ausdifferenzierung und globalen Diffusion der Wirtschaftswissenschaft nach dem Zweiten Weltkrieg erlangt hat: Einführende Lehrbuchliteratur in den Wirtschaftswissenschaften stellt nicht nur eine „überaus ritualisierte Textsorte“ (Hesse 2010: 256) dar, sie bildet auch einen Markt von gigantischem Ausmaß: In den USA sind es jährlich etwa eine Million Studierende, die einführende Veranstaltungen der Wirtschaftswissenschaft belegen (etwa den obligatorischen Kurs Econ 101) und auf diesem Wege mit der entsprechenden Literatur in Berührung kommen (Bartlett 1996: 141). Die Autoren der erfolgreichsten Lehrmaterialien erhalten mitunter Vorschüsse und Honorare, die ansonsten wohl nur im Bereich belletristischer Bestseller üblich sind. Auch wenn - wie später argumentiert - diese Textgattung keinesfalls den State of the Art des Fachs repräsentiert, kann ihr mit Blick auf ihre Deutungsmächtigkeit ein zentraler Platz zugesprochen werden: Insofern weniger als zwei Prozent oben genannter Studierender Wirtschaftswissenschaft als Hauptfach belegen und mit einem Master abschließen, sind Veranstaltungen wie Econ 101 für die große Mehrheit die einzige (formale) wirtschaftswissenschaftliche Ausbildung (Green 2009: 2). Hinzu kommt eine Suggestivkraft der Lehrbücher, die sich gerade nicht auf die Vermittlung komplexer Theorietechniken zu beziehen scheint (oder jedenfalls: nicht darin aufgeht), sondern auf die vorherrschend dargestellte Art und Weise, wie „ordentliches“ und wissenschaftliches Nachdenken über ökonomische Sachzusammenhänge auszusehen habe: Während die formale Präsentation neoklassischer Verfahren (Indifferenzkurven etc.) sich kaum als 
prägend erweisen dürfte, ist damit zu rechnen, dass die offerierten „globalen“ Weichenstellungen und Rahmungen einen bleibenden Eindruck hinterlassen und das Zielpublikum längerfristig beeinflussen. Es sind solche Rahmungen, die eine kritische Betrachtung verdienen (Cohn 2000: 2).

Bereits in Schumpeters klassischer Dogmengeschichte (posthum veröffentlicht in den 1950er Jahren) wurde als "das wichtige Symptom des Erfolgs“ einer Theorierichtung bzw. eines ökonomischen Paradigmas das „Erscheinen einiger erläuternder und einführender Werke“ angegeben (Schumpeter 2009: 1165). Heute dürfte noch mehr gelten, dass deren Inhalt und Präsentationsweise ein guter Indikator dessen ist, welches die in der Disziplin akzeptierten Dogmen und präferierten Themen sind (Ferber/Nelson 2003: 15). Korrelierend mit der ausgeprägten Zentrums-/Peripherie-Differenzierung des Fachs sind die dortigen Lehrbücher wesentlich standardisierter und an einem als gesichert betrachteten Wissenskanon orientiert als beispielsweise in der Soziologie oder in anderen Sozial- und Kulturwissenschaften. Ferber und Nelson, von der Warte feministischer Ökonomik aus gegen die neoklassische Hegemonie opponierend, bezeichnen das Feld der Textbooks denn auch als „impregnable bastion“ (ebd.). So ist es zu erklären, dass sich - ebenfalls ein Unikum der Wirtschaftswissenschaften - nicht nur innerdisziplinäre Protestbewegungen wie die Post-Autistic-Economics formiert haben, sondern ebenfalls Gruppierungen wie die Toxic Textbook Initiative (http://www.toxictextbooks.com), die es sich auf die Fahne geschrieben haben, die am weitesten verbreiteten Lehrmaterialien einer Evaluation und Kritik zu unterziehen und perspektivisch Alternativen auf den Weg zu bringen.

Mein Beitrag soll auf dem Wege empirischer Probebohrungen einige Charakteristika des Genres Textbook Economics herausarbeiten und ansatzweise im Kontext der gesellschaftlichen wie wissenschaftlichen "Großwetterlage“ situieren. Dazu erfolgt eingangs (Teil 2) als Hintergrundfolie ein Abriss zum Feld der Wirtschaftswissenschaft im 20. Jahrhundert, bevor Beispiele aus drei Lehrbüchern diskutiert werden, um ebenso symptomatische wie problematische Argumentationsweisen herauszuarbeiten (Teil 3). Abschließend (Teil 4) werden diese Befunde kontextualisiert und verdichtet sowie offene Forschungsfragen (in Bezug auf Mathematisierung, Performativität sowie die gegenwärtige Wirtschaftskrise) benannt, um am Ende auf die Frage (soziologischer) Alternativen zu sprechen zu kommen.

\section{Eine Hintergrundfolie: Zum Aufstieg und (möglichen) zukünftigen Bedeutungsverlust neoklassischer Ökonomie}

Sozialwissenschaftliche Kritik an der Ökonomik tendiert oftmals dazu, die Disziplin der Wirtschaftswissenschaften als monolithischen Block zu betrachten, als 
dessen Zentrum ein harter, neoklassischer Paradigmenkern identifiziert wird, der das Fach schrittweise okkupiert habe und das gesamte Feld seit langer Zeit konkurrenzlos dominiert. Im Folgenden werden einige Argumente geliefert, weshalb ein solches Interpretationsraster als übersimplifizierend betrachtet werden muss, was nicht bedeutet, die These neoklassischer Hegemonie zu negieren, aber sie etwas anders zu situieren. Ein knapper historischer Abriss soll dazu dienen, die darauffolgenden Überlegungen zu den Textbook Economics zu kontextualisieren.

Die Konstellation ökonomischer Wissenschaftskultur, die gegenwärtig das Feld wie auch die öffentliche Wahrnehmung der Wirtschaftswissenschaft dominiert, aber an den Forschungsfronten einer schleichenden Erosion ausgesetzt zu sein scheint, kann nicht allein mit der marginalistischen Wende und den daran angekoppelten allgemeinen Gleichgewichtstheorien identifiziert werden. Zwar kann tatsächlich für das 20. Jahrhundert zunächst ein Übergang From Interwar Pluralism to Postwar Neoclassicism (Morgan/Rutherford 1998) konstatiert werden: War die Struktur der wirtschaftswissenschaftlichen Disziplin zu Beginn des Jahrhunderts durch ein multipolares Nebeneinander mehrerer akademisch institutionalisierter Theorietraditionen charakterisiert, ${ }^{1}$ zeigte sich spätestens nach dem Zweiten Weltkrieg ein ganz anderes Bild: Die nun maßgeblich von den USA als neuem Zentrum von Wissensproduktion global diffundierende ökonomische Wissenschaftskultur zeigt deutliche monoparadigmatische Einschläge sowie eine Verengung der als valide geltenden wissenschaftlichen Zugriffsweisen. Das Zustandekommen dieser Konstellation lässt sich allerdings keinesfalls nur durch die in der Zwischenzeit erfolgte konsequente Mathematisierung der neoklassischen Theorie (durch Samuelson, Hicks und andere) erklären, sondern erfordert mindestens die Berücksichtigung zweier weiterer Faktoren, nämlich: a) die zeitgleich erfolgte Revolutionierung der Volkswirtschaftlichen Gesamtrechnung sowie b) die Verbindung von Neoklassik und Volkswirtschaftlicher Gesamtrechnung durch die vor allem in der Cowles Commission entstandene Ökonometrie, die eine Anwendung statistischer Techniken auf die von der neuen Gesamtrechnung gelieferten Daten im Rahmen der gerade erst mathematisierten Modelle ermöglichte (vgl. dazu Backhouse 2002: 237ff.). Erst das bei genauerem Hinsehen vielfach kontingente Zusammentreffen dieser Faktoren, zu dem noch eine Reihe sozialstruktureller und anwendungsbezogener Aspekte in Rechnung

1 Für Deutschland kann neben einem Aufgreifen der klassischen politischen Ökonomie vor allem an die Stränge der Historischen Schule erinnert werden, für die USA an den ökonomischen Institutionalismus. 
gestellt werden müssen, ${ }^{2}$ hat zügig zur Konsolidierung beigetragen. Zusammen mit jener relativ stabilen Wachstumskonstellation kapitalistischer Entwicklung nach 1945 (Fordismus) emergierte ein wissenschaftliches Selbstbewusstsein, das bei Fourcade (2009: 85) mit folgenden Worten beschrieben wurde: „The economy had been turned into a 'thing' whose behavior could be described (through national accounts), modeled into equations, tested, predicted, and acted upon".

Ich möchte nur auf zwei einschlägige Resultate verweisen: Zum ersten kann der überraschend zügige Niedergang des älteren ökonomischen Institutionalismus in der Nachkriegszeit damit erklärt werden, dass das dortige Projekt einer quantitativ fundierten Wirtschaftswissenschaft durch den gemeinsamen Aufstieg mathematisierter Wirtschaftstheorie und Ökonometrie „obsolet" gemacht wurde (Fourcade 2009: 83f.). Galt die neoklassische Theorie bis in die 1930er Jahre hinein noch weithin als begrenzter, empirieferner Ansatz und der Institutionalismus als ein Theorieprogramm, das statistische und ökonometrische Kompetenz für sich beanspruchen konnte, so wurde durch die oben genannte erfolgreiche Verkopplung eine neuartige Konstellation geschaffen, in der das Programm des Institutionalismus fortan als Measurement Without Theory kritisiert werden konnte: Die Econometric Society erreichte eine Umstrukturierung der Kräfteverhältnisse, indem quantitative Forschung von einem „Partner“ des Institutionalismus zu einem der rein mathematischen Wirtschaftstheorie gemacht wurde (Yonay 1998: 188). Als zweites Beispiel kann die Assimilierung der Keynesschen Theorie angeführt werden, für die sich der Terminus der neoklassischen Synthese eingebürgert hat. Keynes' Ansatz bestand originär darin, eine Reihe von funktionalen Relationen zwischen makroökonomischen Aggregatgrößen aufzustellen sowie nur schwer zu modellierende Faktoren wie Unsicherheit und Erwartungsbildung prominent zu berücksichtigen. Die spätestens mit Patinkin (1965) kodifizierten, in den 1960er und 1970er Jahren zum Standardrüstzeug fast aller Ökonomen avancierten Keynesian Economics hingegen können als Spezialfall neoklassischer Ökonomie interpretiert werden: Die makroökonomischen Aussagensysteme wurden im Optimierungshandeln rationaler Agenten fundiert, das Theoriedesign formalisiert (Hicks' IS/LM-Modell), Aspekte die sich der Axiomatisierung entzogen wurden eskamotiert.

Inwieweit bis heute im Mainstream der Wirtschaftswissenschaft von einer bruchlosen Dominanz dieser neoklassischen Wissenschaftskultur gesprochen werden kann, ist unklar. Ein Blick auf aktuelle Stimmen aus den Bereichen Dogmengeschichte bzw. Social Studies of Science kann auf zahlreiche Indizien

2 Hier ist u.a. zu denken an: die Weltwirtschaftskrise der 1920er und 1930er Jahre, die Politik des New Deal sowie an den großfl̈chigen Einsatz ökonomischer Expertise im Verlauf von Planungsaktivitäten im Zweiten Weltkrieg (vgl. Mirowski 2002). 
verweisen, wonach deren hegemonialer Stellenwert seit den 1970er Jahren ein Stück weit in Auflösung begriffen ist. Colander et. al (2004) haben hierfür den Terminus eines Changing Face of Mainstream Economics geprägt, Davis (2008) nennt hieran anschließend vor allem die Bereiche Game Theory, Experimental Economics, Behavioral Economics, Neuroeconomics und Non-Linear Complexity Economics als Forschungsstränge, die zwar mit einigen fundamentalen Prämissen und Axiomen der Neoklassik brechen, aber zugleich mehr oder weniger akzeptierte Theorievarianten des Mainstreams darstellen. Anders als bei der tradierten Corona heterodoxer Ansätze, die seitens der institutionalisierten Fachvertreter zumeist mit Nichtbeachtung gestraft wurden, stimmen viele Kommentare in der Vermutung überein, wonach die genannten „gemäßigten Abweichler" für die Entwicklung besonders entscheidend sind: Sie verfügen über das Potential, die Grenzen der Disziplin nachhaltig zu verschieben und neuen Ideen zum Durchbruch zu verhelfen (Backhouse 2004: 269). An anderer Stelle diagnostiziert Colander (2003: 23) eine Verschiebung des in der Makroökonomie - als primäre Beratungsinstanz der Wirtschaftspolitik - anzutreffenden wissenschaftlichen Selbstverständnisses. Die Makroökonomie nehme danach mehrheitlich von der noch in den 1970er Jahren festgeschriebenen Sichtweise Abstand, dass formale Theorien unmittelbar für wirtschaftspolitische Handlungsanweisungen einsetzbar sind. Stattdessen würden zunehmend pragmatische Trial-and-Error-Verfahren Verwendung finden, die nur noch mittelbar durch fundamentale Theoreme und Axiome angeleitet sind. Colander (ebd.: 23) hat hierfür die Bezeichnung einer „muddling-through vision“" geprägt, die - verglichen mit vormaligen sozialtechnologischen Erwartungshaltungen - einen bescheideneren Anspruch anzeigen soll.

Es ist zum jetzigen Zeitpunkt schwer zu eruieren, inwiefern die skizzierten Befunde über ein Changing Face zutreffend sind, relativ sicher - und der Fortgang wird dies exemplarisch zeigen - kann davon ausgegangen werden, dass sich etwaige Relevanzverschiebungen gerade auf dem Terrain der Textbooks (noch) nicht zureichend abbilden. Hier scheint nach wie vor ein Narrativ von Wissenschaftskultur tradiert zu werden, das die Ökonomik als im Kern monoparadigmatische Disziplin auffasst und darstellt, als einen Korpus nomothetischen Wissens, der als unhinterfragtes Fundament eine Reihe von Abstraktionen und Theorietechniken voraussetzt. Kritische Beobachter haben genau an dieser Diskrepanz angesetzt, so wenn bei Klamer (1990: 131) moniert wird, dass die Darstellungen in der Lehrbuchliteratur fälschlicherweise den Eindruck eines Konsenses unter den Ökonomen erwecken würden, um die Disziplin „harten“ wissenschaftlichen Standards entsprechend aufzubereiten. Bei Klamer, McCloskey und Ziliak (2007: 2) wird ähnlich argumentiert, die Wirtschaftswissenschaften stellten faktisch eine Pluralität von heterogenen Konversationen dar, die Lehrbücher seien demgegenüber bestürzend homogen gearbeitet. 


\section{Symptomatische Argumentationsstrategien und Darstellungsweisen im Feld der Textbook Economics - Einige Fallbeispiele}

Als Untersuchungsgegenstand aus eigenem Recht wurde das Feld bis dato eher selten analysiert. Vorherrschend sind Beiträge, die entsprechendes Textmaterial quantitativ abgetastet haben um auf dieser Grundlage beispielsweise die ungenügende Repräsentation von Frauen und Minderheiten aufzuzeigen (vgl. Feiner/Morgan 1987, Robson 2001). Der hier präsentierte Zugriff kann zwar als Ergänzung zu solchen Unternehmungen verstanden werden, das Augenmerk liegt allerdings anders. Die im Folgenden durchgeführten Stichproben können und sollen nicht mit Anspruch auf strenge Repräsentativität auftreten, es geht darum, Indizien für ebenso symptomatische wie problematische Aussageordnungen zusammenzutragen. Des weiteren liegt die Fokussierung nicht primär auf der Frage der immanenten kognitiven Validität des präsentierten Wissens - dass beispielsweise dargestellte Ableitungszusammenhänge fehlerhaft sind, ist eher unwahrscheinlich -, sondern auf der Art und Weise ihrer Rabmung. Dies betrifft zum einen ganz allgemein den Faktor der narrativen Einbettung von als Fakten präsentierten Sachverhalten: „Facts make sense only insofar as they are arranged in some narrative order; moreover, these narratives always are conveyed through metaphors" (Horwitz 1999: 154). Spezifischer betrifft es aber auch die Frage der Referenz, also den Gegenstandsbezug der entwickelten theoretischen Abstraktionen. Die Selektion der herangezogenen Bücher erfolgte nach pragmatischen Gesichtspunkten. Mit Mankiws und Taylors (2008) Grundzüge der Volkswirtschaftslehre wird eines der am weitesten verbreiteten Lehrbücher berücksichtigt, das Buch gilt aufgrund seiner relativ sparsamen Verwendung mathematischer Formeln als gleichermaßen wirklichkeitsnah wie zugänglich (hat aber, auch aufgrund der Tätigkeit Mankiws als Vorsitzender des Rates der Wirtschaftsberater unter George W. Bush in besonderer Weise Kritik auf sich gezogen). Starrs (1997) Einführung in die allgemeine Gleichgewichtstheorie wurde gewählt, weil hier jener Theoriebestand einführend erläutert wird, der von vielen Beobachtern als harter Kern des ökonomischen Mainstreams interpretiert wird. Schließlich wird mit Paschkes (2007) Grundlagen der Volkswirtschaftslehre - anschaulich dargestellt ein drittes Buch herangezogen, das sich als besonders preiswertes Lehrmaterial ebenfalls einer großen Auflagenstärke erfreut und zudem von einem Autor verfasst wurde, der keine akademische Position inne hat, sondern sich aufs Schreiben entsprechender Unterrichtsmaterialien verlegt hat.

Mankiw und Taylors (2008: VII) Lehrbuch beginnt mit einer Art Anekdote - in den Vorbemerkungen für den Lehrenden wird folgende Rahmung präsentiert: „Ich war in einer Familie aufgewachsen, die am Esstisch oft über Politik diskutierte. Das Pro und Contra zu verschiedenartigen Lösungen gesellschaftlicher 
Probleme entfachte hitzige Debatten. In der Schule jedoch fühlte ich mich zu den naturwissenschaftlichen Fächern hingezogen. Während mir die Politikwissenschaften unscharf, beliebig und subjektiv erschienen, waren die Naturwissenschaften analytisch, systematisch und objektiv. Während sich die politische Debatte endlos im Kreise drehte, erzielten die Naturwissenschaften Fortschritte. Meine Anfängervorlesung über 'Principles of Economics' öffnete mir jedoch die Augen für eine neue Art der Betrachtung und des Denkens. Die Volkswirtschaftslehre verbindet die Stärken von Politik- und Naturwissenschaften. Sie ist im Wortsinne eine Sozialwissenschaft. Ihr Hauptgegenstand ist die Gesellschaft - wie Menschen über ihre Lebensführung entscheiden und wie sie zusammenwirken. Gleichwohl geht sie leidenschaftslos wie eine Naturwissenschaft zu Werke. Durch die Anwendung naturwissenschaftlicher Methoden auf politische Fragen sucht die Volkswirtschaftslehre bei den grundlegenden Herausforderungen voranzukommen, denen alle Gesellschaften gegenüberstehen“. Die Wirtschaftswissenschaft erhält in diesem Narrativ eine Sonderstellung innerhalb der Sozialwissenschaften zugesprochen, weil sie durch die Anwendung naturwissenschaftlicher Methoden objektive Antworten auf soziale Phänomene und Probleme zu geben vermöge, wohingegen die anderen, als subjektiv charakterisierten Sozialwissenschaften genau dies nicht leisten, sondern unscharf und beliebig bleiben.

Diese Grundsituierung, die als Widerhall des Samuelsonschen (2005: 5) Diktums von der Wirtschaftswissenschaft als „queen of the social sciences“ gelesen werden kann, wird in Kapitel 2 über Volkswirtschaftliches Denken wieder aufgegriffen und fortgeführt, wenn Mankiw und Taylor sich zum methodologischen Selbstverständnis der Wirtschaftswissenschaft äußern: Ökonomen „betreiben die Erforschung der Volkswirtschaft in ziemlich derselben Weise, wie ein Physiker die Materie und ein Biologe das Leben untersucht: Sie entwerfen Theorien, sammeln Daten und versuchen dann aufgrund der Daten, ihre Theorie zu bestätigen oder zu verwerfen" (ebd.: 23). Im Fortgang wird die Einheit beider Wissenschaftstypen zwar ein Stück weit relativiert, so wenn vermerkt wird, dass Ökonomen nicht auf Erkenntnismittel wie Reagenzgläser oder Teleskope zurückgreifen können, um in analoger Weise wie in den Naturwissenschaften Experimente durchzuführen. Als Ersatz dazu verweisen Mankiw und Taylor auf eine als allgemein und disziplinenübergreifend vorgestellte „wissenschaftliche Methode“, die sie als Abfolge „Beobachtung, Theorie und erneute Beobachtung“ (ebd.: 24) kennzeichnen.

Es ist allerdings grundsätzlich zu hinterfragen, ob das damit proklamierte Methodenideal der Realität entspricht. Der Methodologe und Dogmenhistoriker Blaug (1997: XIII) hat ein immer wieder anzutreffendes Auseinanderfallen von methodologischen Ansprüchen und tatsächlichen Praktiken mit dem Terminus des ,innocuous falsificationism “ (harmloser oder unverfänglicher Falsifikationismus) versehen, worin sich der Vorwurf artikuliert, dass die Mehrzahl der (Main- 
stream-)Ökonomen zwar ein an der empirischen Überprüfung von Hypothesen orientiertes Forschungsverständnis proklamiert, tatsächlich aber ganz anders verfährt. Blaug (ebd.: 169) bezeichnet den vorherrschenden Wissenschaftsmodus der neoklassischen Tradition als endlose Formalisierung rein logischer Problemstellungen ohne jeglichen Bezug auf die Produktion falsifizierbarer Theoreme über tatsächliches ökonomisches Verhalten. Es ist zwar zutreffend, dass Mankiws und Taylors Lehrbuch - anders als viele hier gänzlich unsensible Lehrmaterialien - an zahlreichen Stellen auf die Grenzen generalisierter Modelle und Gesetzesaussagen verweist. ${ }^{3}$ Trotzdem folgt der präsentierte Modus der Wissensgenerierung keinesfalls der proklamierten Logik von „Beobachtung, Theorie und erneute(r) Beobachtung“. Dies könnte exemplarisch gezeigt werden an der Darstellung von Märkten (ebd.: 71ff.) oder bezüglich der Betrachtung von Geld und Preisen (ebd.: 705ff.). In allen Fällen ist es so, dass die basalen Theorietechniken als sakrosankt vorausgesetzt werden, also gerade keiner empirischen Überprüfung ausgesetzt werden, sondern allein notwendige Modifikationen und den Modellen inhärente Restriktionen erörtert werden. Diese Problematik, die ein Grundproblem der Textbook Economics im Modus der Neoklassik bildet, kann anhand des zweiten ausgewählten Lehrbuchs deutlich konkretisiert werden.

Der erste inhaltliche Teil in Starrs (1997) Einführung in die allgemeine Gleichgewichtstheorie, ${ }^{4}$ Concept and history of general equilibrium theory (ebd.: 3ff.), setzt mit einer Unterscheidung von Theorien partiellen und allgemeinen Gleichgewichts ein; die Überlegenheit Letzterer wird plausibilisiert durch Verweis auf empirisch leicht feststellbare Interdependenzen zwischen einzelnen Märkten: Im Falle des US-amerikanischen Automobilmarkts des Jahres 1974 haben Preisveränderungen auf einem anderem Markt (hier: eine Vervierfachung des

3 So heißt es im Kapitel zu den Grenzbereichen der Mikroökonomik, wo Bereiche wie die Verhaltensökonomik oder das Problem der asymmetrischen Informationen skizzenhaft behandelt werden, abschließend: „Wenn es einen verbindenden Gedanken zu all den hier behandelten Themen gibt, dann den, dass das Leben schwierig ist. Die Informationen sind unvollständig, die Regierung ist unvollkommen und die Menschen ebenso" (Mankiw, Taylor 2008: 556). Den Abschnitt über Gewerkschaften und kollektive Lohnverhandlungen resümierend wird festgestellt: „Einigkeit darüber, ob Gewerkschaften gut oder schlecht für eine Volkswirtschaft sind, besteht unter Nationalökonomen nicht. Wie viele Institutionen sind auch die Gewerkschaften unter bestimmten Bedingungen nützlich und bei anderen Gegebenheiten nachteilig für die Gesellschaft“ (ebd.: 697).

4 Auch dieses Buch enthält vor den materialen Teilen ein rahmendes Narrativ, in diesem Fall (vgl. ebd.: XIX) wird dem Leser über eine universitär abgehaltene Geburtstagsfeier berichtet, wo "the fortieth anniversary of one of the greatest achievements of modern economic theory: the mathematical theory of general economic equilibrium " zelebriert wurde. Im hier nicht komplett wiedergegebenen Passus wird auf die Elemente Tradition, wissenschaftliche Reputation und Aktualität verwiesen. 
Benzinpreises) die Nachfrage nach sparsameren Autos aus Japan schlagartig ansteigen lassen und zugleich einen Rückgang der Nachfrage nach amerikanischen Modellen bewirkt. Die allgemeine Gleichgewichtstheorie wird als ein Theorieprogramm beschrieben, dem zwei Leistungen inhärent seien: Sie habe sich erstens als essentiell in der Beschreibung der Effizienz und Stabilität des Marktmechanismus erwiesen sowie zweitens darin, der ökonomischen Analyse ein logisches Fundament zu liefern. Beide Attribute werden miteinander verkoppelt, um die allgemeine Gleichgewichtstheorie als rigorose Einlösung fundamentaler wirtschaftswissenschaftlicher Erkenntnisinteressen auszuweisen: Sie habe die Basis für die entscheidenden Innovationen der modernen ökonomischen Theorie gelegt und eine vollständige, streng mathematische Bestätigung tradierter ökonomischer Sichtweisen geliefert (ebd.). Der Traditionsbezug ebenso wie die Überlegenheit des Theorieprogramms wird im anschließenden Abschnitt History of general equilibrium theory (ebd.: 7ff.) verstärkt, indem - bevor der innertheoretische Fortgang des gleichgewichtstheoretischen Paradigmas (einsetzend mit Walras und endend mit Arrow und Debreu) skizziert wird - verschiedenen klassischen Ökonomen ein ähnlich gelagertes Erkenntnisinteresse zugeschrieben wird: Ökonomen des 19. Jahrhunderts, wie Ricardo, Mill, Marx und Jevons hätten allesamt über ein Konzept stabiler Gleichgewichtstendenzen in der Ökonomie verfügt und hätten die Bedeutung der Interaktion zwischen Märkten erkannt, seien aber noch nicht in der Lage gewesen, diese Einsichten mathematisch zu formalisieren (ebd.). ${ }^{5}$ An Ort und Stelle fehlt auch nicht der Hinweis auf die prominente Smithsche Metapher der unsichtbaren Hand, als deren präzise wissenschaftliche Einlösung die Gleichgewichtstheorie präsentiert wird. Die einleitend strukturierende Dramaturgie findet in Summary and conclusion (ebd.: 237ff.) einen Abschluss, indem das Fazit gezogen wird: „There it is in modern mathematical form - just what Adam Smith (1776) would have said. The competitive market can work to effectively decentralize efficient allocation decisions."

In dieser Situierung findet sich eine mehrfache Amalgamierung heterogener Erkenntnisleistungen, die theoretisch nicht gedeckt ist: Die am Beispiel der Interaktion mehrerer Märkte demonstrierte Überlegenheit allgemeiner gegenüber partieller Theorien des Gleichgewichts sowie dann die Proklamation der Gleichgewichtstheorie als Einlösung des Programms der Smithschen Metapher suggeriert, dass es sich bei ihr um eine Analyse empirischer Märkte handeln würde, um eine analytische Abbildung der realen Prozesse ökonomischer Synthesis.

5 Die Inklusion von Marx in diese Ahnenreihe dürfte kaum mit dessen eigenem Selbstverständnis in Einklang zu bringen sein, wofür die Zentralität krisentheoretischer Argumentationsstränge in der Kritik der politischen Ökonomie ein deutlicher Beleg sein dürfte. 
Die Verweise auf durch das freie Spiel der Marktkräfte generierte wirtschaftliche Optima staffiert das Forschungsprogramm sowie den Objektbereich mit einer zusätzlichen, normativen Komponente aus, indem die unter den artifiziellen Modellbedingungen deduzierbaren Gleichgewichtszustände als sozial wünschenswerte Zustände ausgewiesen werden. Bei Sugden (2002: 114) wird zwischen begrifflicher Exploration (conceptual exploration) und empirischer Theoriebildung (empirical theorizing) unterschieden. Begriffliche Exploration - und als solche muss die gesamte Tradition gleichgewichtstheoretischen Denkens klassifiziert werden - untersucht die internen Eigenschaften von Modellen, ohne hierbei die Beziehung von Modellwelt und empirischer Realität zu berücksichtigen. Dies in Rechnung gestellt kann geschlussfolgert werden, dass die Verkopplung gleichgewichtstheoretischer Gleichungssysteme mit Prozessen realer ökonomischer Ausgleichsprozesse eine trügerische ist: Das Theorem der unsichtbaren Hand ist entweder eine deskriptive oder eine normativ-evaluative Aussage über die Struktur vollständigen Wettbewerbs, wohingegen das Theoriedesign der allgemeinen Gleichgewichtstheorie nicht beansprucht (bzw. nicht beanspruchen kann), wie auch immer geartete Aussagen über deren Realität zu treffen (Blaug 1997: 163). Es hat als solches keinen empirischen Inhalt, bei seinen Prämissen handelt es sich - in der Theoriesprache der klassischen Kantischen Epistemologie ausgedrückt - nicht um synthetische Urteile a priori, sondern um analytische Urteile: „In einem solchen Urteil ist nichts aus Erfahrung enthalten, es zergliedert nur einen Begriff, um diesen universell auf die Erfahrung anwenden zu können" (Jonas 1964: 110). Man muss es nicht kategorisch ablehnen, solcherlei Forschungen zu betreiben, es kann jedenfalls nicht ausgeschlossen werden, dass durch das Ausloten der Eigenschaften von Modellwelten interessante Erkenntnisse ermöglicht werden. Problematisch erscheint in jedem Fall die das Lehrbuch durchziehende Prämisse, wonach die allgemeine Gleichgewichtstheorie uns in direkter Weise das Operieren des Marktmechanismus sehen lasse (Starr 1997: 13), mit der unterstellt wird, dass die offerierten Abstraktionen - wenigstens im Prinzip - den realen Marktmechanismen entsprechen.

Am Beispiel von Paschkes (2007) Lehrbuch soll vor allem die Verschlingung von positiver Darstellung und politischer Wertung dargestellt werden. In den letzten Kapiteln dieses Buches werden zunächst Bedingungen gesamtwirtschaftlichen Gleichgewichts erläutert, bevor im Abschnitt Konjunkturelle Impulse durch die Wirtschaftspolitik nachfrageorientierte und angebotsorientierte Wirtschaftspolitik einander gegenübergestellt werden. Die Ausführungen sind durchgehend mit Wertungen durchsetzt, die allerdings nicht von der positiven Darstellung abgesetzt (und sei es nur durch die Verwendung von Konjunktiven), sondern in diese eingeflochten werden. Den Auftakt machen folgende Ausführungen: „Die deutsche Wirtschaftspolitik hat sich seit den 60er Jahren immer wieder auch nachfrageo- 
rientiert gezeigt. Ein Ergebnis ist ein Schuldenstand der gesamten öffentlichen Haushalte 2005 in Deutschland von rund EUR 1,4 Billionen. [...] Darüber hinaus stecken Deutschland und auch andere europäische Staaten in einer tiefen strukturellen Krise. Viele Strukturen sind verkrustet und die Wettbewerbsbedingungen dienen dem Erhalt des Status Quo. Sie fördern allerdings nicht die dringend benötigte Innovationskraft am Standort Deutschland“ (ebd.: 285f.). Präsentiert wird eine exklusive Verkopplung des Themas der Staatsverschuldung (die de facto diverseste Ursachen haben kann ${ }^{6}$ mit nachfrageorientierter Wirtschaftspolitik, woraufhin eine Aussage folgt, die populäre, politischen Debatten entlehnte Vokabeln wie die der Verkrustung oder der Innovationskraft bemüht. Anschließend erfolgt eine Historisierung keynesiansisch inspirierter Wirtschaftspolitik, sie sei „heute und in der früheren Vergangenheit für die westlichen Industrienationen das falsche Mittel für die Wirtschaftspolitik“, dies sei „die herrschende Meinung führender Ökonomen“ und es gäbe „nur noch wenige Ausnahmen, die den Keynesianismus akzeptieren und noch weniger, die ihn aktiv anwenden wollen“ (ebd.: 287). Im Anschluss an die Bemerkung, dass die Keynessche Theorie „aus einer vollkommen anderen Situation heraus entstanden ist" (starke konjunkturelle Unterbeschäftigung in Verbindung mit Deflation zu Zeiten der Great Depression), die „zum Glück in unseren heutigen Republiken nicht gegeben“ sei, wird erneut konkretistisch für Gegenstimmen Partei ergriffen: „Vor diesem Hintergrund ist eine Neuorientierung der Wirtschaftspolitik, nach den positiven Elementen der Vereinigten Staaten und Großbritanniens zu erhoffen“ (ebenda: 287ff.). Schon bevor der eigene Abschnitt zur angebotsorientierten Wirtschaftspolitik begonnen hat, ist das Feld auf eine Weise vorformatiert, die Wirtschaftspolitik auf eine Auswahl aus nur zwei möglichen Alternativen eingrenzt und auch hier schon ein eindeutiges Votum ausgegeben hat.

Der folgende Darstellungsgang präsentiert neben einigen theorietechnischen Erläuterungen abermals politische Wertungen, wobei nun auch konkretere Maßnahmen Erwähnung finden: Zuvor bereits im Allgemeinen adressierte „veraltete Strukturen“, die „aufgebrochen und dereguliert werden“ müssen, werden nun direkt identifiziert, hierunter fallen beispielsweise „die traditionellen Mitbestimmungsrechte der Arbeitnehmer aus den Montanunion Zeiten“ (ebd.: 292). „(S)ogenannte Flächentarifverträge, die weitgehende Bindung besitzen, [müssen] durch die Einführung weiterer Öffnungsklauseln stärker flexibilisiert werden und durch Ergänzungstarifverträge zur Standortsicherung betriebsnäher gestaltet werden“ (ebd.: 310f.) Mit Blick auf die Reform sozialer Sicherungssysteme wird

6 Zum Beispiel großzügige Steuersenkungen für Unternehmen und Bezieher hoher Einkommen. 
empfohlen: „Wer leisten kann, sollte dies tun und gegebenenfalls hierzu auch von der Gesellschaft durch die Schaffung von Anreizen mehr oder weniger gezwungen werden“" (ebd.: 311). Der gesamte Abschnitt erweckt den Eindruck, lediglich politische Stellungnahmen einer ganz bestimmten Couleur an den Mann/die Frau bringen zu wollen, für die das mitgeführte technische Instrumentarium lediglich eine Art Beiwerk oder Feigenblatt darstellt. Damit korrespondiert auch der den Band abschließende letzte Absatz, wo das angebotsaffirmative Narrativ mit einer prognostischen Verlängerung in die Zukunft ausstaffiert wird: „Zum Schluss sei noch vermerkt, dass sich auch bereits etwas in Deutschland getan hat. Ein Bericht der Weltbankgruppe vergleicht die (De-)Regulierungen in 175 Ländern. Deutschland rangiert hier insgesamt auf Platz 21, unverändert gegenüber dem Vorjahr. Seit dem ersten Bericht 2004 haben Bürokratieabbau bei der Unternehmensgründung und auch leichte Deregulierungen auf dem Arbeitsmarkt stattgefunden. Der Weg an die Spitze oder mindestens näher heran erscheint allerdings entweder weit oder lang. Der nächste Bericht kann mit Spannung erwartet werden" (ebd.: 317). Die Weltbankgruppe wird hier nicht als interessengeleiteter Akteur markiert, sondern als neutrale Instanz im Allgemeininteresse, die dort publizierte Deregulierungsskala wird nicht hinterfragt oder mit alternativen, möglicherweise andere Kriterien ansetzenden Rankings konfrontiert, sondern als objektiver Maßstab angelegt.

\section{Fazit und Ausblick: Die Wirtschaftswissenschaften und ihre Kritik im Kontext}

Ich habe dafür argumentiert, wirtschaftswissenschaftliche Lehrbuchliteratur einer kritischen sozialwissenschaftlichen Evaluierung zu unterziehen, und hierfür Beispiele geliefert. Ausgehend von der Überlegung, dass Lehrbüchern einerseits eine überaus wichtige Bedeutung bei der Repräsentation und Prägekraft der Wirtschaftswissenschaft nach außen (Wirtschaftspolitik) wie bei ihrer Identität nach innen (wissenschaftliche Sozialisation) zukommt, aber andererseits nicht davon ausgegangen werden kann, dass dort der Forschungsstand der Disziplin (die Research Frontiers) adäquat widergespiegelt wird, sollte das Forschungsfeld als Thema aus eigenem Recht situiert werden. Es ist nicht deckungsgleich mit der allgemeinen Evaluation und Kritik der Wirtschaftswissenschaften, sondern erfordert eigene Zugriffsweisen und Bewertungsmaßstäbe. ${ }^{7}$ Die herangezogenen

7 Dieser Befund lässt die gerade seitens heterodoxer Kritiker oftmals praktizierten Verfahren, die Dominanz der Neoklassik durch eine Kritik ihrer Lehrbuchversionen anzugreifen, als problematisch erscheinen (was hier aber nicht weiter verfolgt werden kann). 
Textbeispiele haben sich auf ausgewählte Aspekte beschränkt, die sich mir als besonders augenscheinlich dargestellt haben. Bei Mankiw lag mein Fokus auf den von ökonomischer Seite oftmals proklamierten Bezugnahmen aufdie erfolgreichen Naturwissenschaften und damit (vermeintlich) implizierte Methodenideale, bei Starr ging es um die Problematik von Modellwelten und empirischer Realität und bei Paschke wurde das Phänomen der Amalgamierung der positiven Darstellung von Theorietechniken und wirtschaftspolitischen Werturteilen deutlich gemacht. Natürlich könnten diese Punkte leicht durch weitere Beispiele ergänzt werden. Durchgängig angetroffen wird beispielsweise eine Bestimmung der Wirtschaftswissenschaft als Knappheitslehre, die allzu vermittlungslos auf vermeintlich natürlich vorliegende Bedürfnisstrukturen und ähnlich gelagerte Anthropologismen Bezug nimmt. Man mag von solchen Setzungen halten, was man will, es wäre mindestens einzufordern, die Differenzen von entsprechenden Generalisierungen und der auf bestimmten Sozialstrukturen aufruhenden modernen kapitalistischen Ökonomie explizit zu markieren. Zudem wird einem monoparadigmatischen Bild der Disziplin Vorschub geleistet, das erstens kaum der Realität entspricht und zweitens bereits im Vorfeld dafür sorgt, die Wissenschaft der Wirtschaft auf eine ganz bestimmte Sichtweise festzulegen. Würden andere Theorieprogramme als Alternative und mögliche Korrekturinstanz in den Textbook Economics wenigstens genannt, würde der selektive Charakter der Darstellung sichtbar werden. In den postkeynesianischen Forschungsrichtungen etwa wird Wirtschaftswissenschaft grundsätzlich nicht als Verteilungslehre knapper Ressourcen gerahmt, sondern als Analyse, in welcher Weise ökonomische Systeme in der Lage sind, ihren „Output“ im Zeitverlauf zu erhöhen, wobei insbesondere auf die Produktion und Distribution des Mehrprodukts abgestellt wird (Arestis 1996: 114). Wiederum andere Faktoren müssten genannt werden, wenn Forschungstraditionen wie der Neo-Marxismus oder die feministische Ökonomie miteinbezogen werden würden. In allen diesen und vielen weiteren Fällen geht es nicht nur um Detailkorrekturen am Theoriebestand oder um periphere Interpretationsunterschiede bezüglich vermeintlicher Fakten, sondern darum, was überhaupt als Wirtschaft intelligibel wird.

Bei einem interdisziplinären Vergleich verwundert zudem die mal implizit bleibende, mal explizit herausgestellte, aber durchgängig anzutreffende Affirmation des Objektbereichs „Marktwirtschaft“, die gesamtwissenschaftlich eine Anomalie darstellen dürfte (VertreterInnen von Physik oder Soziologie mag als déformation professionelle eine Faszination gegenüber ihren jeweiligen Realitätsausschnitten eigen sein, die aber kaum mit einem Habitus in eins fallen dürfte, wonach die physikalische Realität oder das Soziale kategorial mit positiven Attributen zu versehen wären). Natürlich ist die im vorliegenden Text offerierte Argumentationsweise nicht unangreifbar. VertreterInnen der Mainstream-Ökonomik 
könnten beispielsweise einwenden, dass die Fixierung auf von der Komplexität der Realität abstrahierende Modellannahmen den Darstellungsnotwenigkeiten eines als kumulativ verstandenen Wissenschaftstypus geschuldet und somit ein Stück weit unumgänglich ist. Ich möchte dies nicht kategorisch bestreiten, aber anmerken, dass in diesem Fall eine weit größere Sorgfalt geboten wäre, den jeweiligen Abstraktionsgrad der herangezogenen Modelle zu explizieren. Gerade der in der Lehrbuchliteratur omnipräsente Rekurs auf einprägsame und einfache Beispiele kann die notwendige kritische Distanz von Modellwelt und Empirie unterminieren, indem auf unmittelbare Evidenzen rekurriert wird. Hierin dürfte ein Einfallstor liegen für die stetig anzutreffende Amalgamierung von positiver Forschung und krypto-normativen Werturteilen. Eine damit eng verbundene Problematik bezieht sich auf den spezifisch ökonomischen Gegenstandsbezug. Die didaktisch motivierte Verwendung von Beispielen ist natürlich keine Besonderheit der (Mainstream)-Ökonomik, sie findet sich ebenso in den heterodoxen Spektren der Disziplin wie in anderen Wissenschaften und hat jeweils ihr gutes Recht in Prozessen der Wissensvermittlung. Aber anders als bei den Naturwissenschaften hat die Wirtschaftswissenschaft (stärker noch als andere Sozial- und Kulturwissenschaften) unmittelbare soziale und politische Auswirkungen, jede Theorie führt zu irgendeiner Variante von Wirtschaftspolitik und diese wiederum begünstigt bzw. verletzt die Interessen unterschiedlicher Gruppierungen. Gerade der Rekurs auf anschauliche Beispiele könnte eigentlich dazu herausfordern, die jeweils enthaltenen politischen Konnotationen explizit transparent zu machen, um der Zielgruppe der Lehrmaterialien eine reflektierte Urteilsbildung zu ermöglichen. ${ }^{8}$

Bevor abschließend Potenziale und Schwierigkeiten insbesondere soziologischer Alternativen zur dominanten Wirtschaftswissenschaft skizziert werden, möchte ich zumindest noch drei Themenkomplexe nennen, die zwar im weiteren Einzugsbereich des Textes lagen, aber vertieft werden müssten. Dies betrifft erstens den Problemkomplex der Mathematisierung: Die neoklassische Wissenschaftskultur zeichnet sich durch einen Grad an Formalisierung, Axiomatisierung und Mathematisierung aus, für den es im Bereich der anderen Sozial- und Kulturwissenschaften keine vergleichbaren Beispiele gibt. Selbst wenn die reine Mathematik als Grenzfall wissenschaftssoziologischer Intervention

8 Das Thema der politischen "Imprägniertheit“ jeglicher Wirtschaftstheorie kann hier nicht detailliert entfaltet werden. Zum einen beeinflussen, wie oben vermerkt, basale Theorieentscheidungen bereits das, was überhaupt als Objektbereich „Wirtschaft“ intelligibel wird. Daneben sind natürlich auch handfeste Interessenlagen in Rechnung zu stellen, aber auch Faktoren, die mit der Selektivität der Vergabe von Forschungsmitteln und mit Publikationschancen in Zusammenhang stehen. 
anzusetzen ist, insofern dort jene ,interpretative Flexibilität“ (Heintz 2000: 12) nicht gegeben sein mag, die bei anderen Wissenschaften als Einsatzpunkt für sozialwissenschaftliche Korrekturen (des jeweils vertretenen wissenschaftlichen Selbstverständnisses) fungiert, habe ich anlässlich der Frage der Relationierung von Modellbildung und empirischer Realität auf einen einschlägigen Sachverhalt aufmerksam gemacht: Im Fall der mathematischen Ökonomik bleibt die Frage der Referenz oftmals prekär. Das mathematische Ideal, „Palaver in Rechnen zu überführen", verdankt sich der damit gestifteten Trennung von Syntax und Semantik: „Im Gegensatz zu einem alltäglichen Gespräch, bei dem wir nicht davon abstrahieren können, was ein Wort bedeutet, vollzieht sich in der Mathematik die Manipulation der Zeichen losgelöst von deren Interpretation“ (ebd.). Es gibt gute Gründe für die Annahme, dass eine Trennung von Syntax und Semantik auf dem Terrain der Ökonomik (wie bei jeder empirischen Wissenschaft) nicht sinnvoll durchzuhalten ist, sondern deren stetige Relationierung virulent bleibt. Kritische Ökonomen haben immer wieder darauf hingewiesen, dass es sich auf dem Feld der Wirtschaftswissenschaft niemals „um rein mathematische Größenbeziehungen “ handelt, „sondern um Beziehungen zwischen realen, empirischen Größen, und da muß man sich beim mathematischen Ausdruck immer fragen: Was wird ausgedrückt?" (Amonn 1927: 124). Damit entwickelte mathematische Verfahren (z.B. die Differentialrechnung) sinnvoll angewendet werden können, muss der Gegenstand eine Reihe keineswegs selbstverständlicher Eigenschaften aufweisen. Mir scheint, dass es hier regelmäßig schon am Problembewusstsein mangelt, mathematische Techniken werden seitens vieler Ökonomen in gleicher Weise verwendet, wie man als Abiturient die auswendig gelernten Ableitungsregeln anwendet, nämlich ohne darauf zu reflektieren, was diese mathematisch implizieren.'

Zum zweiten wurde in jüngster Zeit vor allem seitens der Social Studies of Finance eine vieldiskutierte These hinsichtlich der Performativität ökonomischer Theorien zur Diskussion gestellt: Es wurde die Auffassung vertreten, die Wirtschaftswissenschaft beschreibe nicht primär eine außer ihr liegende Realität, sondern affiziere ihren Objektbereich selbst, und dies zum Teil sehr massiv. Die dortigen empirischen Fallbeispiele beziehen sich nicht von ungefähr schwerpunktmäßig auf jene Bereiche der Finanzwirtschaft, für die plausibel argumentiert werden kann, dass theoretische Prämissen „in die alltäglichen Arbeitsprozesse und Geschäftsroutinen eingepflanzt“ (Vogl 2011: 110) werden,

9 So wird bei nahezu allen Funktionen unterstellt, dass man sie ableiten kann. Alle gängigen „Grenz“-Konzepte (Grenznutzen, Grenzertrag) gehen von dieser Voraussetzung aus, es wird aber nicht gefragt, ob eine differenzierbare Funktion eine gute Annäherung an die ökonomische Wirklichkeit darstellt. 
wofür eine für den praktischen Einsatz im Derivatehandel gedachte Formel von Black und Scholes das Standardbeispiel abgibt. Die vorliegende Analyse der Textbook Economics verschiebt das Erkenntnisinteresse eher auf die Bedeutung mannigfacher Vermittlungsinstanzen, denen gegenüber starke Performativitätsannahmen als Spezialfall erscheinen. Es ist unwahrscheinlich, von der Ebene der lehrbuchartigen Darstellung der Wirtschaft kausale, eindeutig dingfest zu machende Einflüsse auf den Objektbereich zu erwarten, gleichwohl darf deren Wirkmächtigkeit nicht unterschätzt werden. Gerade Popularisierung kann als Medium interpretiert werden, durch das jenes an den Forschungsfronten generierte Wissen in die Gesamtgesellschaft diffundiert und handlungswirksam wird. Hierbei rückt die Frage der kognitiven Validität in die zweite Reihe, von primärem Interesse erweisen sich die Mittel, aufgrund derer ein solcher Transfer vonstatten geht. ${ }^{10}$

Drittens schließlich soll wenigstens grob abgesteckt werden, ob im Anschluss an die jüngste Wirtschafts- und Finanzkrise mit verstärkter Selbstkritik innerhalb des ökonomischen Mainstreams zu rechnen ist. Im Zuge der Krise wurde in einschlägigen überregionalen Tageszeitungen zeitweise intensiv über eine sich ebenfalls offenbarende Krise der Wirtschaftswissenschaft gestritten (eine Fallstudie dazu liegt vor bei Pahl 2011). Eine Voraussetzung für beschleunigte Umstrukturierungen der Ökonomik würde die Frage darstellen, ob das prognostische Versagen der Zunft innerhalb des Fachs auch tatsächlich als Krise wahrgenommen wird, wofür es gegenwärtig nur wenige Indizien gibt. Grundsätzlich ist davon auszugehen, dass das moderne, disziplinär ausdifferenzierte und an Berufschancen ausgerichtete Fach der Wirtschaftswissenschaft eine erhebliche Brechungsstärke besitzt, externe Resonanzen und Turbulenzen zu filtern. Mir scheint vor diesem Hintergrund die Annahme plausibel, dass Veränderungspotential vor allem seitens jener Forschungsstränge zu erwarten ist, die oben mit dem Terminus des Changing Face versehen wurden, weil hier aus dem Fach selbst heraus Korrekturbedarf angemeldet wird.

Was könnte die Soziologie angesichts dieser Diagnosen offerieren? Der Gesamtbestand soziologischer Beiträge zur Wirtschaft der Gesellschaft lässt sich idealtypisch entlang zweier Pole aufspannen: Auf der einen Seite rangieren Theorieprogramme - prominent im Gefolge der Marxschen Kritik der politischen Ökonomie, aber auch im Umfeld der Theorie sozialer Systeme - die auf die Singularität der modernen Wirtschaft und ihrer Bewegungslogik abstellen. Hier werden Alternativen zur Neoklassik angeboten, die aber grundsätzlich mit

10 Als Beispiel könnte an die Diskussion der Humankapitaltheorie bei Foucault (2006) erinnert werden, die plastisch macht, dass Grundcharakteristika der marginalistischen Wende erst über den Weg der Popularisierung breitenwirksam geworden sind. 
der dortigen These eines gesetzesmäßig strukturierten Objekts übereinstimmen, das sich von anderen Bereichen des Sozialen kategorial unterscheidet. Auf der anderen Seite befinden sich die Eingaben einer New Economic Sociology, die auf die soziale Einbettung ökonomischer Sachverhalte abstellt, sowie die erst in den Kinderschuhen steckenden poststrukturalistischen Beiträge zur Wirtschaft. Diese widersprechen der ökonomischen Neoklassik nicht mit alternativen, prinzipiell ebenfalls nomothetisch ausgerichteten Gegenprogrammen, sondern möchten beiderlei Wissenschaftskulturen einer Grundlagenkritik unterziehen und Prämissen ökonomischer Gesetzesmäßigkeit der Tendenz nach als Chimäre ausweisen. ${ }^{11}$ Ich möchte nicht pauschal für oder gegen einen dieser Stränge Partei ergreifen, sondern auf eine Diagnose aufmerksam machen, die Marion Fourcade (2010: 68) aufgestellt hat. Beim Vergleich der Deutungsmächtigkeit von Wirtschaftswissenschaft und Wirtschaftssoziologie stellt sie fest, dass beide Disziplinen sehr unterschiedliche strukturelle Positionen zueinander und zur Politik einnehmen. Die Wirtschaftswissenschaft ist traditionell in Staat, Unternehmen und internationalen Organisationen weitaus einflussreicher, Ökonomen verfügen nicht nur über größere monetäre Ressourcen, sie haben auch eine Kultur des „fix-it" etabliert. Durch diese werden nicht nur mögliche Inhalte selektiert, sondern bereits die grundsätzlichen Erwartungshaltungen an die Form sozialwissenschaftlicher Beratung vorstrukturiert. Die Soziologie ist in diesen Bereichen deutlich im Hintertreffen, wobei Fourcade insbesondere die Makroebene wirtschaftspolitischer Beratung, wo politisch folgereiche Entscheidungen und Weichenstellungen verhandelt werden, als Terrain fern jeglicher soziologischer Expertise und Interventionsmöglichkeit markiert.

Hieraus kann ein Fazit abgeleitet werden, das die obige Differenz soziologischer Paradigmen ein Stück weit durchkreuzt: dass soziologische Alternativen in stärkerem Maß makroökonomische Kompetenz erlangen müssen, wenn sie gegenüber der Mainstream-Ökonomik Terrain gutmachen möchten. Dazu ist es allerdings notwendig, nicht nur alternative Paradigmen und Zugriffsweisen zu erarbeiten, sondern die „Großwetterlage“ einer Revision zu unterwerfen. Das Ansinnen nach grundsätzlich anders gelagerten Beschreibungen wird keinen durchschlagenden Erfolg zeitigen, solange sowohl die dominanten Erwartungsstrukturen im Bereich wirtschaftspolitischer Expertise (nach sozialtechnologischer Beratung) als auch

11 Dies zeigt am deutlichsten ein aktueller Text von Beckert (2011), wo unter dem Titel Was sind Preise? vorhandene soziologische und wirtschaftswissenschaftlich-heterodoxe Erkenntnisse eruiert werden. Ein Resultat besteht darin, dass der Nexus von Angebot und Nachfrage lediglich einen Faktor darstellt, durch den Preisbildungsprozesse affiziert werden, die exklusive Fokussierung des ökonomischen Mainstreams auf dieses Moment wird so als selektive Zugriffsweise sichtbar. 
jene im Bereich universitärer Ausbildung (nach streng formalisierbaren Wissensordnungen) nicht dekonstruiert oder wenigstens nach haltig irritiert werden. Gefordert ist hierzu eine systematischere sozialwissenschaftliche Evaluation und Kritik der Ökonomik, die bis dato eine Blindstelle darstellt, wozu das vorliegende Themenheft allerdings einen überfälligen Beitrag leistet.

\section{Literatur}

Amonn, Alfred (1927): Objekt und Grundbegriffe der theoretischen Nationalökonomie. Leipzig, Wien.

Arestis, Philip (1996): Post-Keynesian economics: towards coherence. In: Cambridge Journal of Economics, Jg. 20: 111-135.

Backhouse, Roger E. (2004): A Suggestion for Clarifying the Study of Dissent in Economics. In: Journal of the History of Economic Thought, Jg. 26, H. 2: 261-271.

Backhouse, Roger E. (2002): The Penguin history of economics. London.

Bartlett, Robin L. (1996): Discovering Diversity in Introductory Economics. In: Journal of Economic Perspectives, Jg. 10: 141-153.

Beckert, Jens (2011): Where Do Prices Come From? Sociological Approaches to Price Formation. Köln. (MPIfG Discussion Paper, 11/3). Online verfügbar unter http://www.mpifg.de/pu/ mpifg_dp/dp11-3.pdf, zuletzt geprüft am 09.06.2011.

Blaug, Mark (1997): The methodology of economics. Or how economists explain. Cambridge.

Cohn, Steve (2000): Telling Other Stories: Heterodox Critiques of Neoclassical Micro Principles Texts. Tufts University. Medford MA. (Global Development and Environment Institute Working Paper, 00-06). Online verfügbar unter http://ase.tufts.edu/gdae/publications/working_papers/ TellingOtherStories.pdf, zuletzt geprüft am 06.05.2011.

Colander, David (2003): Post Walrasian Macro Policy and the Economics of Muddling Through. In: International Journal of Political Economy, Jg. 33, H. 2: 17-35.

Colander, David C.; Holt, Richard P. F.; Rosser, John Barkley (2004): The changingface of economics. Conversations with cutting edge economists. Ann Arbor.

Davis, John B. (2008): The turn in recent economics and return of orthodoxy. In: Cambridge Journal of Economics, Jg. 32: 349-366.

Feiner, Susan F.; Morgan, Barbara A. (1987): Women and Minorities in Introductory Economic Textbooks: 1974 to 1984. In: The Journal of Economic Education, Jg. 18, H. 4: 376-392.

Ferber, Marianne A.; Nelson, Julie A. (2003): Beyond Economic Man, Ten Years Later. In: Ferber, Marianne Abeles; Nelson, Julie A. (Hg.): Feminist economics today. Beyond economic man. Chicago: $1-31$.

Foucault, Michel (2006): Die Geburt der Biopolitik. Vorlesung am Collège de France 1978 - 1979. Frankfurt am Main.

Fourcade, Marion (2010): Interview with Marion Fourcade. In: Economic Sociology - the european electronic newsletter, Jg. 11, H. 3: 66-68.

Fourcade, Marion (2009): Economists and societies. Discipline and profession in the United States, Britain, and France, 1890s to 1990s. Princeton.

Green, Tom L. (2009): Introductory Economics Textbooks: What do they teach about sustainability? Paper submitted to the 8th International Conference of the European Society for Ecological Economics. Online verfügbar unter http://www.esee2009.si/papers/Green\%20-\%20Introductory\%20Economics\%20Textbooks.pdf. 
Heintz, Bettina (2000): Die Innenwelt der Mathematik. Zur Kultur und Praxis einer beweisenden Disziplin. Wien.

Hesse, Jan-Otmar (2010): Wirtschaft als Wissenschaft. Die Volkswirtschaftslehre in der frühen Bundesrepublik. Frankfurt am Main.

Horwitz, Howard (1999): The Toggling Sensibility. Formalism, Self-Consciousness, and the Improvement of Economics. In: Woodmansee, Martha; Osteen, Mark (Hg.): The new economic criticism. Studies at the intersection of literature and economics. London: 150-174.

Jonas, Friedrich (1964): Das Selbstverständnis der ökonomischen Theorie. Berlin.

Klamer, Arjo (1990): The Textbook Presentation of Economic Discourse. In: Samuels, Warren J. (Hg.): Economics as discourse. An analysis of the language of economists. Boston: 129-154.

Klamer, Arjo; McCloskey, Deirdre N.; Ziliak, Stephen (2007): Is There Life after Samuelson's Economics? Changing the Textbooks. In: Post-Autistic Economics Review, H. 42: 2-7.

Mankiw, Nicholas Gregory; Taylor, Mark P. (2008): Grundzüge der Volkswirtschaftslehre. 4., überarb. und erw. Aufl. Stuttgart.

Mirowski, Philip (2002): Machine dreams. Economics becomes a cyborg science. Cambridge.

Morgan, M. S.; Rutherford, M. (Hg.) (1998): From Interwar Pluralism to Postwar Neoclassicism. Durham, NC.

Nasar, Sylvia (1995): A Hard Act to Follow? Here Goes. In: The New York Times, 14.03.1995. Online verfügbar unter http://www.nytimes.com/1995/03/14/business/a-hard-act-to-followhere-goes.html, zuletzt geprüft am 05.01.2011.

Pahl, Hanno (2011): Die Wirtschaftswissenschaften in der Krise. Vom massenmedialen Diskurs zu einer Wissenssoziologie der Wirtschaftswissenschaften, in: Schweizerische Zeitschrift für Soziologie, Sonderheft The Global Economic Crisis: Perceptions and Impacts (in press).

Patinkin, Don (1965): Money, Interest and Prices: An Integration of Monetary and Value Theory. New York.

Robson, Denise (2001): Women and Minorities in Economics Textbooks: Are They Being Adequately Represented? In: The Journal of Economic Education, Jg. 32, H. 2: 186-191.

Samuelson, Paul Anthony (2005): Economics. 18. ed., internat. ed., Boston.

Schumpeter, Joseph A. (2009): Geschichte der ökonomischen Analyse. Göttingen.

Starr, Ross M. (1997): General equilibrium theory. An introduction. Cambridge.

Sugden, Robert (2002): Credible worlds: the status of theoretical models in economics. In: Mäki, Uskali (Hg.): Fact and fiction in economics. Models, realism, and social construction. Cambridge: 107-136.

Yonay, Yuval Peretz (1998): The struggle over the soul of economics. Institutionalist and neoclassical economists in America between the wars. Princeton.

Vogl, Joseph (2010): Das Gespenst des Kapitals. Zürich. 